3-15-2008

\title{
Elastically Induced Coexistence of Surface Reconstructions
}

Jessica E. Bickel

Cleveland State University, j.e.bickel@csuohio.edu

Normand A. Modine

Sandia National Laboratories

Chris Pearson

University of Michigan-Flint

Joanna Mirecki Millunchick

University of Michigan

Follow this and additional works at: https://engagedscholarship.csuohio.edu/sciphysics_facpub

Part of the Physics Commons

How does access to this work benefit you? Let us know!

\section{Repository Citation}

Bickel, Jessica E.; Modine, Normand A.; Pearson, Chris; and Millunchick, Joanna Mirecki, "Elastically Induced Coexistence of Surface Reconstructions" (2008). Physics Faculty Publications. 194.

https://engagedscholarship.csuohio.edu/sciphysics_facpub/194

This Article is brought to you for free and open access by the Physics Department at EngagedScholarship@CSU. It has been accepted for inclusion in Physics Faculty Publications by an authorized administrator of

EngagedScholarship@CSU. For more information, please contact library.es@csuohio.edu. 


\title{
Elastically induced coexistence of surface reconstructions
}

\author{
Jessica E. Bickel, ${ }^{1}$ Normand A. Modine,${ }^{2}$ Chris Pearson, ${ }^{3}$ and Joanna Mirecki Millunchick ${ }^{1, *}$ \\ ${ }^{1}$ Department of Materials Science and Engineering, University of Michigan, Ann Arbor, Michigan 48109, USA \\ ${ }^{2}$ Sandia National Laboratories, Albuquerque, New Mexico 87185, USA \\ ${ }^{3}$ Department of Computer Science, Engineering Science, and Physics, The University of Michigan-Flint, Flint, Michigan 48502, USA
}

(Received 26 July 2007; revised manuscript received 20 December 2007; published 6 March 2008)

\begin{abstract}
Scanning tunneling microscopy of Sb-capped GaAs shows the coexistence of different surface reconstructions. The majority of the surface consists of an $\alpha 2(2 \times 4)$ reconstruction typically observed for GaAs $(001)$ surfaces. At step edges, an $\alpha(4 \times 3)$ reconstruction, common for $\mathrm{GaSb}(001)$, is observed. We argue that strain couples the surface reconstruction to the film morphology. Density functional theory calculations show that the $(2 \times 4)$ reconstruction is stabilized in GaSb films when the lattice parameter is constrained to that of GaAs, as happens in the middle of a terrace, while the $(4 \times 3)$ reconstruction is stabilized when the lattice parameter is allowed to relax toward that of GaSb at step edges. This result confirms the importance of elastic relaxation in the coexistence of surface reconstructions.
\end{abstract}

DOI: $10.1103 /$ PhysRevB.77.125308

PACS number(s): 68.55.-a, 68.35.B-, 68.35.Gy, 68.37.Hk

\section{INTRODUCTION}

Understanding surface reconstructions remains an important, yet challenging endeavor in many materials systems ranging from oxides to compound semiconductors. Reconstructions have a significant influence on epitaxial film growth, the resulting nano- and microscale structure, and on properties such as catalysis. ${ }^{1}$ Surface reconstructions form in order to reduce the energy of a cleaved surface. The stability of a reconstruction is determined by competition between the chemical energy due to the formation of new covalent bonds, the electrostatic energy associated with the rearrangement of electrons within the bonds and dangling bonds of the surface, and the strain energy introduced by displacing atoms from their bulk lattice positions. In systems combining components with different lattice parameters, strain can play additional roles. ${ }^{2}$

Strain gives rise to many well known growth phenomena. Lattice mismatch strain, the strain that arises due to differences in the lattice parameter of the substrate and film, greatly influences growth as large mismatch strains result in a transition from two-dimensional (2D) to three-dimensional (3D) growth for many systems including InAs $/ \mathrm{GaAs}^{3}$ and $\mathrm{GaSb} / \mathrm{GaAs} .{ }^{4}$ Localized atomic size mismatch strain, the strain that arises due to differences in bond lengths in alloy films, can lead to ordering, ${ }^{5}$ phase separation in the form of lateral composition modulation, ${ }^{6,7}$ and changes in surface reconstruction. ${ }^{2}$ Based on theoretical calculations, it has been proposed that lattice mismatch strain may also influence the stability of surface reconstructions. ${ }^{8}$ However, direct evidence of this effect has been limited. An externally applied strain has been shown to induce a preferred dimer orientation in Si films, ${ }^{9}$ and Ge heteroepitaxy on Si has been shown to induce periodic missing dimer rows in the $(2 \times 1)$ reconstruction ${ }^{10}$ though each of these results shows only a small change in a stable reconstruction as a function of strain, rather than a large change in reconstruction. Other work on Ge heteroepitaxy on Si with a Sb surfactant layer suggests that lattice strain induced by the inclusion of the $\mathrm{Sb}$ induces an alternate reconstruction in thin films. ${ }^{11}$
This paper presents a study of thin films of $\mathrm{Sb}$ below the critical thickness for 3D growth, deposited on $\operatorname{GaAs}(001)-(2 \times 4)$ and immediately quenched, to examine the atomistic growth mechanisms of strained GaSb. The resulting films exhibit a mixed surface reconstruction consisting of an $\alpha 2(2 \times 4)$ reconstruction, not commonly seen in bulk $\mathrm{GaSb}$, and an $\alpha(4 \times 3)$ reconstruction, common to many III-Sb systems. In agreement with the experimental data, density functional theory (DFT) studies of surface reconstruction stability as a function of strain show that when this ultrathin GaSb layer is constrained to the GaAs lattice parameter, the $\alpha 2(2 \times 4)$ reconstruction possesses a lower free energy relative to the $\alpha(4 \times 3)$. However, at the terrace edges, where the film may relax elastically, approaching that of bulk GaSb, the $\alpha(4 \times 3)$ is stabilized. This coupling between coexisting reconstructions and morphology provides strong evidence for the dependence of surface reconstruction on lattice mismatch strain.

\section{METHODS AND RESULTS}

\section{A. Experimental methods}

Samples were grown by molecular beam epitaxy using solid source $\mathrm{Ga}$ and valved-cracker As and $\mathrm{Sb}$ sources. Growth rates were calibrated using reflection high-energy electron diffraction (RHEED) oscillations. The samples were grown on $\mathrm{GaAs}(001)$ substrates that were prepared by heating between $620 \leqslant T \leqslant 640{ }^{\circ} \mathrm{C}$ under an $\mathrm{As}_{4}$ overpressure until the surface oxide desorbed. The temperature was then reduced to $T=600{ }^{\circ} \mathrm{C}$, and a $0.5 \mu \mathrm{m}$ thick GaAs buffer layer was grown at a rate $R_{\mathrm{Ga}}=0.30$ monolayers per second (ML/s) under an As flux of $R_{\mathrm{As}}=1.20 \mathrm{ML} / \mathrm{s}$. Prior to $\mathrm{Sb}$ deposition, the sample temperature was decreased to $T=525{ }^{\circ} \mathrm{C}$ while still maintaining a $(2 \times 4)$ reconstruction. Sb films of various thicknesses $0.25 \leqslant h \leqslant 1.7 \mathrm{ML}$ were grown at a $\mathrm{Sb}$ flux of $R_{\mathrm{Sb}}=0.36 \mathrm{ML} / \mathrm{s}$. Since $\mathrm{Sb}$ is a group $\mathrm{V}$ element and the growth takes place at high temperature, it is expected that the $\mathrm{Sb}$ replaces As on the GaAs surface, forming ultrathin layers of $\mathrm{GaSb}$ at these growth conditions. Despite the large lattice 

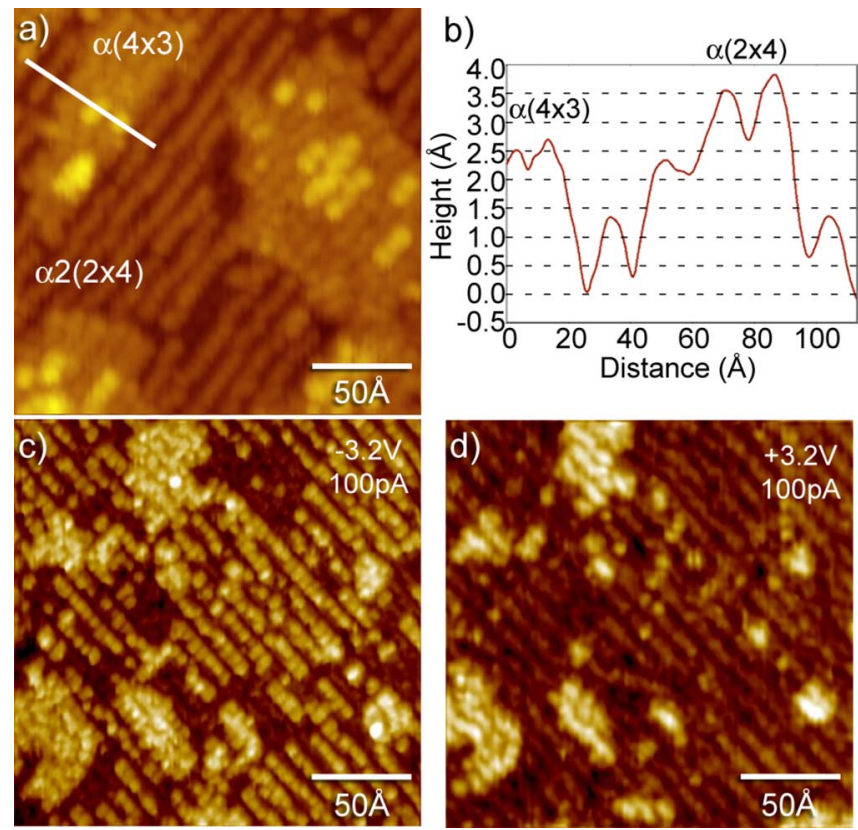

FIG. 1. (Color online) (a) Atomic resolution STM image of $h$ $=1.7 \mathrm{ML} \mathrm{Sb} / \mathrm{GaAs}$ grown at $T=525{ }^{\circ} \mathrm{C}$ and imaged at $-4.3 \mathrm{~V}$ and $100 \mathrm{pA}$. Areas of $\alpha 2(2 \times 4)$ and $\alpha(4 \times 3)$ are labeled. (b) Height profile of the line in (a) with $\alpha 2(2 \times 4)$ and $\alpha(4 \times 3)$ domains labeled. (c) Filled state image and (d) empty state image of $h=0.8$ $\mathrm{ML} \mathrm{Sb} / \mathrm{GaAs}$ grown at $T=525{ }^{\circ} \mathrm{C}$, taken at $V_{\text {bias }}= \pm 3.2 \mathrm{~V}$ and $100 \mathrm{pA}$.

mismatch between GaSb and GaAs (7\%), the resulting films are planar according to both RHEED and scanning tunneling microscopy (STM). This indicates that the critical thickness for $3 \mathrm{D}$ island formation was not reached under these growth conditions. At the conclusion of growth, the samples were immediately quenched to $T=200{ }^{\circ} \mathrm{C}$ by cutting the power to the sample heater, and were then removed from the chamber to cool to room temperature. Samples were characterized in vacuo by STM, with images taken at a tunneling current of $0.1 \mathrm{nA}$ and a bias voltage of $-3<V<-4.5$ for filled state images or $+2.5<V<+3.5$ for empty state images.

\section{B. Experimental results}

The surface structure of very thin layers of $\mathrm{GaSb} / \mathrm{GaAs}$ is predominately a $(2 \times 4)$ reconstruction. However, a second reconstruction is also observed. Figure 1 shows atomic resolution STM images of a 1.7 ML film of Sb/GaAs [Fig. 1(a)] with a corresponding height profile [Fig. 1(b)], and a dual bias image pair of a $0.8 \mathrm{ML}$ film of $\mathrm{Sb} / \mathrm{GaAs}$ taken at $V_{\text {bias }}=-3.2 \mathrm{~V}$ for filled states [Fig. 1(c)] and $V_{\text {bias }}=+3.2 \mathrm{~V}$ for empty states [Fig. 1(d)]. Two surface reconstructions are seen in the images. The majority of the surface is covered by rows that are spaced 14.6 $\mathrm{\AA}$ apart, as seen in Fig. 1(b). This row spacing is in good agreement with a $4 a$ spacing, where $a=3.94 \AA$ is the in-plane lattice parameter, in agreement with the observed $(2 \times 4)$ RHEED pattern. The $(2 \times 4)$ reconstruction is predominately $\alpha 2(2 \times 4)$, which has been well described, ${ }^{12}$ with one As dimer in the trench and one As dimer in the row. The $(2 \times 4)$ reconstruction has not been

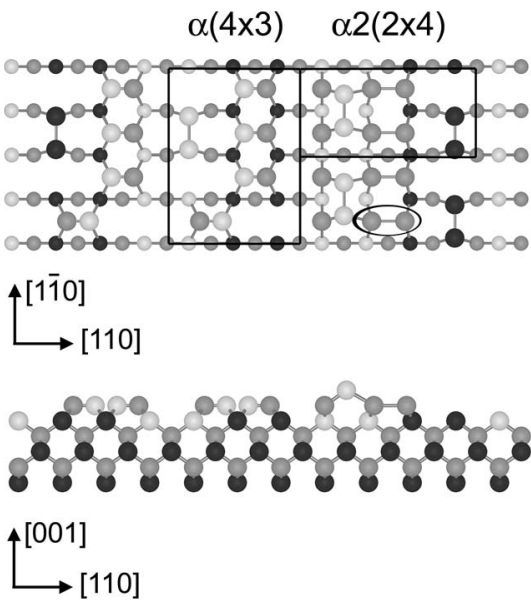

FIG. 2. Schematic diagrams of the mixed surface reconstruction showing the $\alpha(4 \times 3)$ adjacent to an $\alpha 2(2 \times 4)$ from both the surface (top) and side (bottom) views. The circle indicates the cation-cation back bond in the $\alpha 2(2 \times 4)$ reconstruction. Ga, dark gray; As, black; $\mathrm{Sb}$, light gray. Some atoms were removed for clarity.

observed for bulk GaSb, but it has been studied for Sbcapped GaAs both experimentally ${ }^{13-16}$ and theoretically. ${ }^{17,18}$ The $(2 \times 4)$ reconstruction has also been studied as a stable reconstruction of the As-rich GaAsSb alloy and has been proposed as the mechanism for quadruple period ordering in this system. ${ }^{19}$ Other work has analyzed the placement of anions in the GaAsSb surface. ${ }^{20}$ A second surface reconstruction is also observed in this image, primarily along step and 2D island edges. As seen in Fig. 1(b), this reconstruction has a periodic row spacing of $11.2 \AA$, corresponding to $3 a$ spacing, and is $\sim 1.2 \AA$ higher or lower than the adjacent $\alpha 2(2$ $\times 4)$ reconstruction. Thus, there is nominally one atomic layer height difference between this intermediate $\times 3$ reconstruction and the $\alpha 2(2 \times 4)$ reconstructions. The $3 a$ row spacing together with the height differences suggests that this reconstruction is a $(4 \times 3)$ reconstruction commonly observed on bulk GaSb. ${ }^{21}$

The dual bias images [Figs. 1(c) and 1(d)] more specifically indicate the nature of the $(4 \times 3)$ reconstruction. The relative intensity of the two different reconstructions changes with changing bias voltage. The $\alpha 2(2 \times 4)$ is bright in the filled state image and dark in the empty state image, consistent with a surface structure that is comprised of As dimers. The $(4 \times 3)$ reconstruction, on the other hand, is bright in both the filled and empty state images. This suggests that the surface structure is comprised of both cations (which are bright under positive bias) and anions (which are bright under negative bias). Therefore, it is likely that the $(4 \times 3)$ reconstruction observed here is, in fact, the $\alpha(4 \times 3)$ reconstruction, which is comprised of Ga-Sb heterodimers on the surface. $^{21}$

A schematic of the mixed $\alpha 2(2 \times 4)$ and $\alpha(4 \times 3)$ surface reconstructions is displayed in Fig. 2. The $\alpha 2(2 \times 4)$ reconstruction consists of bulk zinc blende structure terminated by a plane of anions, which is covered by $2 / 3 \mathrm{ML}$ of cations, and an anion dimer, which forms a dimer row in the [1 10$]$, separated from adjacent rows by rows of trench anion 
dimers. ${ }^{12}$ The $\alpha(4 \times 3)$ reconstruction consists of bulk zinc blende structure terminated by a plane of anions covered with four anion-cation heterodimers. These dimers also form a row along the $[1 \overline{1} 0]$, with adjacent rows separated by anion trench dimers. However, every fourth surface dimer is displaced in the [110] by one bulk spacing unit, creating a surface kink. The placement of anion-cation heterodimers on a plane of anions introduces an antiphase defect through the anion-anion bond. This model indicates that there should be a height difference of one atomic layer between the $\alpha 2(2$ $\times 4)$ and $\alpha(4 \times 3)$ reconstructions, consistent with the STM height profile in Fig. 1(b). The disorder present in the STM images of the $\alpha(4 \times 3)$ regions can be explained by the fact that the displaced heterodimer, or kink, may be positioned anywhere within the unit cell.

The size of the $2 \mathrm{D}$ islands appears to have a strong influence on the appearance of the $\alpha 2(2 \times 4)$ reconstruction within the islands. For $0.8 \leqslant h \leqslant 1.7 \mathrm{ML} \mathrm{Sb} / \mathrm{GaAs}$, small $2 \mathrm{D}$ islands consist entirely of the $\alpha(4 \times 3)$ reconstruction, as can be seen in the small 2D islands in Figs. 1(c) and 1(d). As the island size increases, the $2 \mathrm{D}$ islands transform to also include patches of the $\alpha 2(2 \times 4)$ reconstruction, as is seen for the large islands in Fig. 1(a). An analysis of many STM images shows that the average island size at which the $\alpha 2(2 \times 4)$ reconstruction appears is $30 \pm 10 \mathrm{~nm}^{2}$.

\section{Computational methods}

The stable surface reconstruction and resulting surface morphology are typically the result of several competing interactions including displacement strain, surface charge neutrality, local chemistry, and chemical potential, all of which are, at least indirectly, temperature dependent. In the case described here, both the $\alpha 2(2 \times 4)$ and the $\alpha(4 \times 3)$ reconstructions exhibit charge neutrality and the correct local chemistry. As they are grown on the same surface, and remain stable upon cooling, the chemical potential is also a constant for these two reconstructions. However, the fact that the $\alpha(4 \times 3)$ reconstruction appears predominately at step edges very strongly suggests that elastic strain relaxation impacts the stability of the reconstructions present on the surface for these very thin strained layers. DFT calculations were used to test this hypothesis. Calculations were performed using the Vienna $a b$ initio simulation package ${ }^{22}$ using ultrasoft pseudopotentials ${ }^{23,24}$ and the local density approximation. ${ }^{25,26}$ Slabs consisted of four bulk layers of GaSb, terminated on the top by the desired reconstruction and on the bottom by pseudohydrogen to charge neutralize the surface. Slabs were separated by at least $11 \AA$ of vacuum to minimize interactions between the top and bottom surfaces, and were relaxed using a $6 \times 3,3 \times 4$, and $3 \times 3$ $k$-point mesh for the $2 \times 4,4 \times 3$, and $4 \times 4$ slabs, respectively. The plane-wave energy cutoff was set at $203.1 \mathrm{eV}$ for all calculations, the electronic temperature was set to $0.025 \mathrm{eV}$, and slabs were relaxed until the total energy was converged to better than $0.1 \mathrm{meV}$.

\section{Computational results}

The resulting surface energies of the $\alpha 2$ and $\beta 2(2 \times 4)$, the $\alpha$ and $\beta(4 \times 3)$, and the $c(4 \times 4)$ reconstructions of $\mathrm{GaSb}$

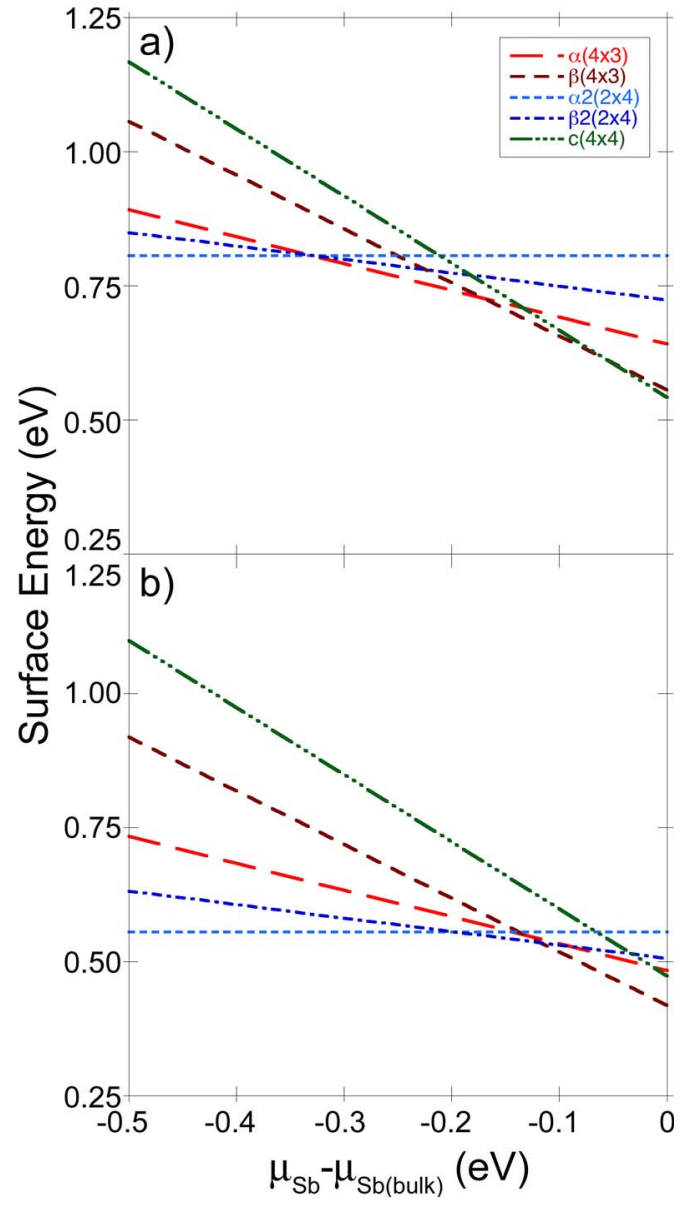

FIG. 3. (Color online) Phase diagram of GaSb at the (a) GaSb and (b) GaAs lattice parameters.

are plotted as a function of $\mathrm{Sb}$ chemical potential in Fig. 3 at both the GaSb [Fig. 3(a)] and GaAs [Fig. 3(b)] lattice parameters. Slabs of pure GaSb were chosen because the surface is assumed to be pure GaSb due to the tendency of $\mathrm{Sb}$ to surface segregate ${ }^{5}$ and also by the fact that $x$-ray studies of Sb-capped GaAs with a $(2 \times 4)$ reconstruction show that $\mathrm{Sb}$ is limited to the surface. ${ }^{20}$ The surface energies are calculated using the method described by Wixom et al. ${ }^{27}$ The $x$ axis is the chemical potential of $\mathrm{Sb}, \mu_{\mathrm{Sb}}$, relative to that of bulk rhombohedral $\mathrm{Sb}, \mu_{\mathrm{Sb}(b u l k)}$. The reconstruction with the lowest energy at a given $\mu_{\mathrm{Sb}}-\mu_{\mathrm{Sb}(b u l k)}$ is predicted to be the stable reconstruction on the surface, with higher energy curves energetically inaccessible. At the GaSb lattice parameter [Fig. 3(a)], the most stable reconstructions with decreasing chemical potential (moving left along the $x$ axis) are the $c(4 \times 4)$, followed by the $\beta(4 \times 3), \alpha(4 \times 3)$, and $\alpha 2(2 \times 4)$. This agrees with the calculations of Righi et al., ${ }^{28}$ which show a $(4 \times 3)$ reconstruction for Sb-rich GaSb and a $\beta 2(2$ $\times 4)$ reconstruction for Ga-rich GaSb. When the GaSb crystal is constrained to the GaAs lattice parameter [Fig. 3(b)], the stability of the different reconstructions changes dramatically. The lines shift relative to each other to the point that the $\alpha(4 \times 3)$ reconstruction is never the lowest line for any chemical potential, and is, thus, excluded as a stable reconstruction at this lattice parameter. Instead, the stable recon- 
structions with decreasing chemical potential are the $\beta(4$ $\times 3)$, followed by the $\beta 2(2 \times 4)$ and $\alpha 2(2 \times 4)$ reconstructions. This is due to changes in the relative energy of the different reconstructions, which can be seen in the $y$-axis intercept point. The changes in relative energy also result in a change in the relative crossover points of the reconstructions. The transition from the $(4 \times 3)$ reconstructions to the $(2 \times 4)$ reconstructions shifts to a higher chemical potential by $\Delta \mu_{\mathrm{Sb}}=0.25 \mathrm{eV}$.

The shift in stability of the surface reconstructions of $\mathrm{GaSb}$ between the GaSb and GaAs lattice parameters is demonstrated by the appearance of both the $\alpha 2(2 \times 4)$ and $\alpha(4 \times 3)$ reconstructions in the $\mathrm{Sb} / \mathrm{GaAs}$ films, with the $\alpha(4 \times 3)$ appearing at step edges where elastic relaxation occurs, and the $\alpha 2(2 \times 4)$ appearing in areas where the lattice parameter is constrained, i.e., within large terraces or near the center of large 2D islands. This mixed reconstruction has not been previously reported, even for Sb-capped GaAs, ${ }^{14-16,29-32}$ likely due to different growth conditions of those experiments, which often included extensive annealing that would decrease the chemical potential, thus further stabilizing the $(2 \times 4)$. A strain induced shift in reconstruction stability has been predicted by $\mathrm{DFT}^{8}$ and suggested as the mechanism behind the atomic structure which develops in the $\mathrm{Sb}: \mathrm{Ge} / \mathrm{Si}(111)$ system. ${ }^{11}$ The work presented here, however, shows that a local variation in strain can produce a coexistence of reconstructions on a single surface.

The mechanism by which the $\alpha 2(2 \times 4)$ surface reconstruction in GaSb is stabilized may be understood by examining the details of the atomic arrangement within the individual reconstructions. Near step edges, the $\mathrm{Sb}$ bond lengths elastically relax toward that of bulk GaSb because they are not laterally constrained by the substrate, and thus may take on the bulk GaSb reconstruction, $\alpha(4 \times 3)$. Away from step edges, the layer is constrained by the substrate, thus inducing the $\alpha 2(2 \times 4)$ reconstruction. This suggests that the $\alpha 2(2$ $\times 4)$ reconstruction can relieve the high compressive strain better than the $\alpha(4 \times 3)$, likely due to the fact that the $\alpha 2(2$ $\times 4)$ has a larger surface corrugation than the $\alpha(4 \times 3)$. As measured in Fig. 1(b), the peak to valley height of the $\alpha 2(2 \times 4)$ is $3.1 \AA$, while that of the $\alpha(4 \times 3)$ is $1.8 \AA$; both are in close agreement with simulation results. By considering other bond lengths and distances between atoms, the mechanism by which the $\alpha 2(2 \times 4)$ relieves stress becomes apparent. Simulation results show that the heterodimer in the $\alpha(4 \times 3)$ reconstruction cannot accommodate the compressive strain induced by being constrained to the GaAs lattice parameter. However, there is a mechanism by which this strain may be relieved in the $\alpha 2(2 \times 4)$ reconstruction. This reconstruction has a cation-cation back bond, which is circled in Fig. 2. This bond is normally in tension. Reducing the lattice parameter to that of GaAs reduces the tension in this bond to compensate exactly for the compressive strain. This explanation is consistent with the experimental observations of the dependence of the reconstruction on 2D island size. Specifically, small 2D islands exhibit solely the $\alpha(4$ $\times 3)$ reconstruction, while $2 \mathrm{D}$ islands greater than a critical size exhibit the $\alpha 2(2 \times 4)$ in the center and $\alpha(4 \times 3)$ at the edges.

\section{CONCLUSIONS}

We have shown the existence of a mixed surface reconstruction for thin layers of $\mathrm{Sb}$ on $\mathrm{GaAs}(001)$ and confirmed the influence of strain in the appearance of this mixed reconstruction. Initially, 2D islands of $\alpha(4 \times 3)$ reconstruction nucleate, and lattice mismatch strain forces a transformation to a mixed reconstruction of $\alpha(4 \times 3)$ and $\alpha 2(2 \times 4)$. The coexistence of these two surface reconstructions on the same sample under the same growth conditions demonstrates that the $\alpha 2(2 \times 4)$ is thermodynamically induced by strain and not due to either temperature or group $\mathrm{V}$ overpressure effects. Thus, strain opens an additional region of stability that allows $\mathrm{GaSb}$ to form the $\alpha 2(2 \times 4)$ reconstruction.

\section{ACKNOWLEDGMENTS}

The authors gratefully acknowledge the support of the Department of Energy, Basic Energy Sciences via Contract No. DE FG02 04 ER46172. Sandia is a multiprogram laboratory operated by Sandia Corporation, a Lockheed Martin Company, for the United States Department of Energy under Contract No. DE-AC04-94AL85000. *joannamm@umich.edu

${ }^{1}$ G. A. Somorjai, Annu. Rev. Phys. Chem. 45, 721 (1994).

${ }^{2}$ J. E. Bickel, N. A. Modine, A. Van der Ven, and J. M. Millunchick Appl. Phys. Lett. 92, 062104 (2008).

${ }^{3}$ D. Leonard, M. Krishnamurthy, C. M. Reaves, S. P. Denbaars, and P. M. Petroff, Appl. Phys. Lett. 63, 3203 (1993).

${ }^{4}$ P. M. Thibado, B. R. Bennett, M. E. Twigg, B. V. Shanabrook, and L. J. Whitman, J. Vac. Sci. Technol. A 14, 885 (1996).

${ }^{5}$ S. Froyen and A. Zunger, Phys. Rev. B 53, 4570 (1996).

${ }^{6}$ C. Dorin and J. M. Millunchick, J. Appl. Phys. 91, 237 (2002).

${ }^{7}$ B. J. Spencer, P. W. Voorhees, and J. Tersoff, Phys. Rev. B 64, 235318 (2001).

${ }^{8}$ C. Ratsch, Phys. Rev. B 63, 161306(R) (2001).

${ }^{9}$ F. K. Men, W. E. Packard, and M. B. Webb, Phys. Rev. Lett. 61,
2469 (1988).

${ }^{10}$ B. Voigtländer and M. Kästner, Phys. Rev. B 60, R5121 (1999).

${ }^{11}$ A. Antons, Y. Cao, B. Voigtländer, K. Schroeder, B. R. Berger, and S. Blügel, Europhys. Lett. 62, R7719 (2003).

${ }^{12}$ C. Ratsch, W. Barvosa-Carter, F. Grosse, J. H. G. Owen, and J. J. Zinck, Phys. Rev. B 62, R7719 (2000).

${ }^{13}$ F. Maeda, Y. Watanabe, T. Scimeca, and M. Oshima, Phys. Rev. B 48, 4956 (1993).

${ }^{14}$ L. Whitman, B. Bennett, E. Kneedler, B. Jonker, and B. B. Shanabrook, Surf. Sci. Lett. 436, L707 (1999).

${ }^{15}$ P. Laukkanen, R. E. Perala, R.-L. Vaara, I. J. Vayrynen, M. Kuzmin, and J. Sadowski, Phys. Rev. B 69, 205323 (2004).

${ }^{16}$ P. Moriarty, P. H. Beton, Y. R. Ma, M. Henini, and D. A. Woolf, Phys. Rev. B 53, R16148 (1996). 
${ }^{17}$ W. G. Schmidt and F. Bechstedt, Phys. Rev. B 55, 13051 (1997).

${ }^{18}$ N. Esser, A. Shkrebtii, U. Resch-Esser, C. Springer, W. Richter, W. Schmidt, F. Bechstedt, and R. D. Sole, Phys. Rev. Lett. 77, 4402 (1996).

${ }^{19}$ I. G. Batyrev, A. G. Norman, S. B. Zhang, and S.-H. Wei, Phys. Rev. Lett. 90, 026102 (2003).

${ }^{20}$ T.-L. Lee and M. J. Bedzyk, Phys. Rev. B 57, R15056 (1998).

${ }^{21}$ W. Barvosa-Carter, A. S. Bracker, J. C. Culbertson, B. Z. Nosho, B. V. Shanabrook, L. J. Whitman, H. Kim, N. A. Modine, and E. Kaxiras, Phys. Rev. Lett. 84, 4649 (2000).

${ }^{22}$ G. Kresse and J. Hafner, Phys. Rev. B 47, 558 (1993); 49, 14251 (1994); G. Kresse and J. Furthmüller, Comput. Mater. Sci. 6, 15 (1996); Phys. Rev. B 54, 11169 (1996).

${ }^{23}$ G. Kresse and J. Hafner, J. Phys.: Condens. Matter 6, 8245 (1994).
${ }^{24}$ D. Vanderbilt, Phys. Rev. B 41, 7892 (1990).

${ }^{25}$ D. M. Ceperley and B. J. Alder, Phys. Rev. Lett. 45, 566 (1980).

${ }^{26}$ J. P. Perdew and A. Zunger, Phys. Rev. B 23, 5048 (1981).

${ }^{27}$ R. R. Wixom, N. A. Modine, and G. B. Stringfellow, Phys. Rev. B 67, 115309 (2003).

${ }^{28}$ M. C. Righi, R. Magri, and C. M. Bertoni, Phys. Rev. B 71, 075323 (2005).

${ }^{29}$ M. Wang, D. Collins, T. McGill, and R. Grant, J. Vac. Sci. Technol. B 11, 1418 (1993).

${ }^{30}$ S. Schmitz, J. Wagner, F. Fuchs, N. Herres, P. Koidl, and J. Ralston, J. Cryst. Growth 150, 858 (1995).

${ }^{31}$ R. M. Feenstra, D. A. Collins, D. Z.-Y. Ting, M. W. Wang, and T. C. McGill, Phys. Rev. Lett. 72, 2749 (1994).

${ }^{32}$ B. Bennett, B. Shanabrook, and M. Twigg, J. Appl. Phys. 85, 2157 (1999). 